\title{
Ring Compound
}

National Cancer Institute

\section{Source}

National Cancer Institute. Ring Compound. NCI Thesaurus. Code C1933.

A cyclic compound. any compound in which the constituent atoms, or any part of them, form a ring. Used mainly in org anic chemistry. SYN closed chain compound, ring compound. 